\title{
Focus particles and negative scope: Both evidence for syntactic integration?
}

\author{
Ira Eberhardt \& Sam Featherston \\ University of Tübingen
}

13th September 2019

\section{Appendix}

\section{Materials for experiment 1 on German}

In each case we give just the forms without focus particles.

1a. Peter hat die Wohnung nicht gekündigt, weil die Miete günstig ist, sondern er hat es nur angedroht.

1b. Peter hat die Wohnung nicht gekündigt, weil die Miete gestiegen ist, sondern weil seine Mitbewohner rauchen.

2a. Philipp hat das Auto nicht verkauft, weil der Motor noch gut lief, sondern er hat es herrichten lassen.

2b. Philipp hat das Auto nicht verkauft, weil der Motor kaputt war, sondern weil der Verbrauch so hoch war.

3a. Markus hat die Schuhe nicht gekauft, weil die Farbe zu grell war, sondern er hat sich ein anderes Paar gesucht.

3b. Markus hat die Schuhe nicht gekauft, weil die Farbe passend war, sondern weil sie bequem waren.

4a. Mark hat seine Schwester nicht besucht, weil die Eltern es verboten haben, sondern er hat ihr einen Brief geschrieben.

4b. Mark hat seine Schwester nicht besucht, weil die Eltern es verlangt haben, sondern weil er sie vermisst hat.

5a. Mandy hat das Foto nicht geschossen, weil das Licht schlecht war, sondern sie hat eine Postkarte gekauft.

5b. Mandy hat das Foto nicht geschossen, weil das Licht sehr gut war, sondern weil die Berge so schön aussahen.

6a. Chris hat das Fitnessstudio nicht gewechselt, weil die Geräte gut waren, sondern er hat seinen Vertrag dort verlängert.

6b. Chris hat das Fitnessstudio nicht gewechselt, weil die Geräte schlecht waren, sondern weil die Anfahrt weit war. 
7a. Kathrin hat den Job nicht angenommen, weil das Gehalt schlecht war, sondern sie hat sich weiter beworben.

7b. Kathrin hat den Job nicht angenommen, weil das Gehalt gut war, sondern weil ihr die Arbeitszeiten gut gepasst haben.

8a. Julia hat das Kleid nicht ausgeliehen, weil die Freundin davon abgeraten hat, sondern sie hat sich für einen Hosenanzug entschieden.

8b. Julia hat das Kleid nicht ausgeliehen, weil die Freundin es empfohlen hat, sondern weil der Schnitt ihr gefallen hat.

\section{Experiment 1 on German: Mean values of ratings and z-scores}

\begin{tabular}{|ll|c|cc|cc|}
\hline Condition & Particle & $n$ & Score & SD & z-score & SD \\
\hline -NegScope -Part & auch & 63 & 23.25 & 6.045 & -0.063 & 0.738 \\
& hauptsächlich & 64 & 22.53 & 5.729 & 0.239 & 0.762 \\
& nur & 64 & 22.03 & 5.688 & -0.316 & 0.647 \\
& vor allem & 62 & 22.98 & 5.467 & -0.172 & 0.655 \\
& Total & 253 & 22.70 & 5.722 & -0.198 & 0.704 \\
\hline -NegScope +Part & auch & 64 & 20.23 & 5.817 & -0.654 & 0.688 \\
& hauptsächlich & 64 & 22.38 & 5.353 & -0.335 & 0.664 \\
& nur & 64 & 20.08 & 6.137 & -0.625 & 0.735 \\
& vor allem & 64 & 21.02 & 5.370 & -0.462 & 0.603 \\
& Total & 256 & 20.93 & 5.718 & -0.519 & 0.683 \\
\hline +NegScope -Part & auch & 64 & 29.89 & 7.211 & 0.884 & 0.874 \\
& hauptsächlich & 64 & 28.05 & 5.573 & 0.701 & 0.772 \\
& nur & 63 & 29.40 & 6.228 & 0.885 & 0.642 \\
& vor allem & 64 & 27.78 & 6.057 & 0.687 & 0.710 \\
& Total & 255 & 28.78 & 6.322 & 0.789 & 0.756 \\
\hline +NegScope +Part & auch & 64 & 20.88 & 5.579 & -0.537 & 0.774 \\
& hauptsächlich & 64 & 22.81 & 4.931 & -0.194 & 0.709 \\
& nur & 64 & 27.55 & 5.369 & 0.564 & 0.744 \\
& vor allem & 64 & 21.86 & 6.094 & -0.356 & 0.909 \\
& Total & 256 & 23.27 & 6.048 & -0.130 & 0.889 \\
\hline
\end{tabular}




\section{Materials for experiment 2 on English}

1a. Peter is staying in his flat. He did not terminate the lease, because the rent is quite low, but he threatened to.

1b. Peter is moving out of his flat. He did not terminate the lease because the rent had gone up but because his flatmates smoke.

2a. Phillip has kept his car. He did not sell it, because the engine was still good, but he had it overhauled.

2b. Phillip does not have his car any more. He did not sell it because the engine was faulty but because the fuel consumption was so high.

3a. Markus decided against the red shoes. He did not buy them, because the colour was too bright, but he bought another pair instead.

3b. Markus has new red shoes. He did not buy them because he liked the colour but because they were comfortable.

4a. Martha has not seen her uncle for ages. She has not visited him, because her parents have forbidden it, but she has secretly called him.

4b. Martha was at her uncle's yesterday. She did not visit him because her parents demanded it, but because she had missed him.

5a. Wendy's house still looks shabby. She has not had it renovated, because the costs would be too high, but she will sell it.

5 b. Wendy's house is shining in new splendour. She has not had it renovated because the tenants demanded it, but because she wants to sell it.

6a. Julia decided not to borrow Hannah's dress. She did not borrow it, because her mother advised her against it, but she decided to wear a suit instead.

6b. Julia looks great in Hannah's dress. She did not borrow it because her mother recommended it but because she liked its style.

7a. Catherine turned down the job at Burger King. She did not take it, because the pay was bad, but she applied for other jobs instead.

7b. Catherine is working at Burger King. She did not take the job because the pay was good but because the working hours suited her.

8a. Tom's flat looks untidy. He did not clean it today, because his parents are not coming for a few weeks, but he went to the beach instead.

8b. Tom's flat looks immaculate. He did not clean it today because his parents are coming but because he always cleans on Saturdays. 
Experiment 2 on English: Mean values of ratings and z-scores

\begin{tabular}{|ll|c|cc|cc|}
\hline Condition & Particle & $n$ & Score & SD & z-score & SD \\
\hline -NegScope -Part & just & 63 & 26.59 & 4.102 & 0.162 & 0.589 \\
& mainly & 64 & 25.23 & 5.994 & 0.012 & 0.697 \\
& purely & 64 & 25.84 & 4.376 & 0.080 & 0.753 \\
& specially & 64 & 25.95 & 5.284 & 0.100 & 0.677 \\
& Total & 255 & 25.90 & 4.992 & 0.088 & 0.680 \\
\hline -NegScope +Part & just & 64 & 24.06 & 5.120 & -0.307 & 0.754 \\
& mainly & 64 & 26.64 & 4.864 & 0.205 & 0.658 \\
& purely & 64 & 25.48 & 6.695 & -0.003 & 0.755 \\
& specially & 64 & 23.94 & 5.404 & -0.361 & 0.683 \\
& Total & 256 & 25.03 & 5.644 & -0.116 & 0.746 \\
\hline +NegScope -Part & just & 64 & 27.84 & 6.234 & 0.507 & 0.710 \\
& mainly & 64 & 28.00 & 5.915 & 0.492 & 0.707 \\
& purely & 64 & 28.27 & 4.501 & 0.647 & 0.545 \\
& specially & 63 & 29.16 & 4.311 & 0.735 & 0.621 \\
& Total & 255 & 28.31 & 5.304 & 0.595 & 0.654 \\
\hline +NegScope +Part & just & 64 & 27.91 & 5.017 & 0.530 & 0.583 \\
& mainly & 64 & 24.03 & 6.259 & -0.294 & 0.863 \\
& purely & 64 & 28.45 & 4.605 & 0.632 & 0.561 \\
& specially & 64 & 25.05 & 6.308 & -0.077 & 0.832 \\
& Total & 256 & 26.36 & 5.870 & 0.197 & 0.819 \\
\hline
\end{tabular}

